\title{
SUM OF THE PERIODIC ZETA-FUNCTION OVER THE NONTRIVIAL ZEROS OF THE RIEMANN ZETA-FUNCTION
}

\author{
RAMŪNAS GARUNKŠTIS AND JUSTAS KALPOKAS
}

\begin{abstract}
We consider the asymptotic of the sum of values of the periodic zeta-function $L(s, \lambda)$ over nontrivial zeros of the Riemann zeta-function $\zeta(s)$. J. Steuding for rational $\lambda$ obtained the main term. Assuming the Generalized Riemann Hypothesis we calculate the next term.
\end{abstract}

\section{INTRODUCTION}

Let $s=\sigma+i t$ denote a complex variable and define $e(z)=e^{2 \pi i z}$. In this paper $T$ always tends to plus infinity and $p$ denotes a prime number. Let $0<\alpha \leq 1$ and $\lambda \in \mathbb{R}$. The Hurwitz zeta-function is given by

$$
\zeta(s, \alpha)=\sum_{n=1}^{\infty} \frac{1}{(n+\alpha)^{s}} \quad(\sigma>1)
$$

and periodic zeta-function by

$$
L(s, \lambda)=\sum_{n=1}^{\infty} \frac{e(\lambda n)}{n^{s}} \quad(\sigma>1)
$$

For $\alpha=\lambda=1$ we get the Riemann zeta-function $\zeta(s)=\zeta(s, 1)=L(s, 1)$. The Riemann hypothesis $(\mathrm{RH})$ asserts that all nontrivial (non-real) zeros of $\zeta(s)$ lie on the critical line $\sigma=1 / 2$. We will need the generalized Riemann hypothesis $(\mathrm{GRH})$. Consider the Dirichlet $L$-function

$$
L(\chi, s)=\sum_{n=1}^{\infty} \frac{\chi(n)}{n^{s}} \quad(\sigma>1),
$$

where $\chi(n)$ is a Dirichlet character. GRH states that in the critical strip $0<\sigma \leq 1$ the function $L(\chi, s)$ has zeros only on the critical line.

Let $\rho=\beta+i \gamma$ denote the nontrivial zero of the Riemann zeta-function. Assuming $\mathrm{RH}$, Fujii [2] showed

$$
\sum_{0<\gamma \leq T} \zeta(\rho, \alpha)=-\frac{T}{2 \pi}\left(\Lambda\left(\frac{1}{\alpha}\right)+L(1,1-\alpha)\right)+O\left(T^{\frac{9}{10}} \log T\right) .
$$

Steuding [6] proved the above formula unconditionally with the error term $O\left(T^{1-c(\log T)^{-2 / 3}}\right)$, where $c$ is an absolute positive constant. Assuming RH, Garunkštis and Steuding [5] improved Fujii's error term to $O\left(T^{\frac{1}{2}+\frac{16}{237}+\varepsilon}\right)$ and, assuming GRH, this error term can be replaced by $O\left(T^{\frac{1}{2}+\varepsilon}\right)$ if $\alpha$ is rational. For $\alpha=1 / 2$ we have

$$
\zeta(s, 1 / 2)=\left(2^{s}-1\right) \zeta(s) .
$$

Thus, on RH, nonreal zeros of $\zeta(s, 1 / 2)$ lie on lines $\sigma=0$ and $\sigma=1 / 2$. In [5] the behavior of sum (1) was investigated when $\alpha=\alpha(T)$ tends to $1 / 2$ and 1 . This inspired a

Date: November 11, 2007.

AMS 1991 subject classification: 11M35, 11M26

Key words and phrases: periodic zeta-function, Riemann zeta-function, sum over zeros 
classification of nontrivial zeros of the Riemann zeta-function dependently on the behavior of zeros of the Hurwitz zeta-function. For more details see [5].

In formula (1) we see the periodic zeta-function. This function is related to the Hurwitz zeta-function by the "functional" equation (Apostol [1], Theorem 12.6)

$$
\zeta(1-s, \alpha)=\frac{\Gamma(s)}{(2 \pi)^{s}}\left(e\left(-\frac{s}{4}\right) L(s, \alpha)+e\left(\frac{s}{4}\right) L(s,-\alpha)\right) .
$$

Let $k$ and $l$ be fixed natural numbers. From Steuding [7] it follows that, for $0<\frac{k}{l}<1$, $(k, l)=1$,

$$
\sum_{0<\gamma \leq T} L\left(\rho, \frac{k}{l}\right)=\left(e\left(\frac{k}{l}\right)-\frac{\mu(l)}{\varphi(l)}\right) \frac{T}{2 \pi} \log \frac{T}{2 \pi}+O(T) .
$$

The aim of this paper is to calculate the $T$ term in the above formula.

For $0<\alpha \leq 1$ we define the generalized Euler constant $\gamma(\alpha)$ by

$$
\sum_{0 \leq n \leq T} \frac{1}{n+\alpha}=\log T+\gamma(\alpha)+O\left(\frac{1}{T}\right) .
$$

We see that $\gamma(1)$ is the usual Euler constant.

For Dirichlet character $\chi \bmod l$ the Gauss sum is defined by

$$
\tau(\chi)=\sum_{a=1}^{l} \chi(a) e\left(\frac{a}{l}\right) .
$$

By $\chi_{0}$ we denote the principal character.

The notation $p^{v}|| l$ means that $p^{v} \mid l$ but $p^{v+1} \nmid l$.

Theorem 1. Assume GRH. Let $k$ and $l$ be fixed natural numbers with $0<\frac{k}{l}<1$ and $(l, k)=1$. Then

$$
\sum_{0<\gamma \leq T} L\left(s, \frac{k}{l}\right)=\left(e\left(\frac{k}{l}\right)-\frac{\mu(l)}{\varphi(l)}\right) \frac{T}{2 \pi} \log \frac{T}{2 \pi}+C\left(\frac{k}{l}\right) \frac{T}{2 \pi}+O\left(T^{9 / 10} \log T\right),
$$

where

$$
\begin{aligned}
C\left(\frac{k}{l}\right) & =-e\left(\frac{k}{l}\right)-\frac{1}{\varphi(l)} \sum_{\substack{\text { mod } l \\
\chi \neq \chi_{0}}} \chi(k) \tau(\bar{\chi}) \frac{L^{\prime}}{L}(1, \chi) \\
& +\frac{\mu(l)}{\varphi(l)}\left(1+\gamma(1)-\gamma\left(\frac{k}{l}\right)+\sum_{p \mid l} \frac{\log p}{p-1}\right)-\sum_{p^{v}|| l} \frac{\log p}{1-p^{-\varphi\left(\frac{l}{p^{v}}\right)}} \sum_{j=1}^{\varphi\left(\frac{l}{p^{v}}\right)} \frac{e\left(p^{j} \frac{k}{l}\right)}{p^{j}}
\end{aligned}
$$

In view of

$$
L(s, 1 / 2)=e(-1 / 2)\left(1-2^{1-s}\right) \zeta(s)
$$

the constant $C\left(\frac{1}{2}\right)$ should be equal to zero. This is indeed, because the sum over characters is empty and $\gamma\left(\frac{1}{2}\right)=2 \log 2+\gamma(1)$.

To prove the theorem we use Fujii's [2] method. We also make use of the sum

$$
\sum_{n=1}^{\infty} \frac{\Lambda(n)-1}{n}=-2 \gamma(1)
$$

To calculate this sum, let us consider the Dirichlet series

$$
\sum_{n=1}^{\infty} \frac{\Lambda(n)-1}{n^{s}}
$$


By the prime number theorem this series converges in the half-plane $\sigma \geq 1$. For $\sigma>1$,

$$
\sum_{n=1}^{\infty} \frac{\Lambda(n)-1}{n^{s}}=-\frac{\zeta^{\prime}}{\zeta}(s)-\zeta(s)
$$

Now we take a limit $s \rightarrow 1$.

\section{Lemmas}

We will use the approximate functional equation for the periodic zeta-function.

Lemma 1. If $0<\lambda \leq 1$, when

$$
L\left(\frac{1}{2}+i T, \lambda\right)=\sum_{1 \leq n \leq \sqrt{\frac{T}{2 \pi}}} \frac{e(n \lambda)}{n^{\frac{1}{2}+i T}}+\left(\frac{T}{2 \pi}\right)^{-T i} e^{T i+\frac{\pi i}{4}} \sum_{0 \leq n \leq \sqrt{\frac{T}{2 \pi}}} \frac{1}{(n+\lambda)^{\frac{1}{2}-i T}}+O\left(T^{-\frac{1}{4}}\right) .
$$

Proof. This lemma is the particular case of the more general approximate functional equation for the Lerch zeta-function $L(\lambda, \alpha, s)$, which can be found in Garunkštis, Laurinčikas, and Steuding [4]. Note that $L(s, \lambda)=e(\lambda) L(\lambda, 1, s)$.

In the proof of Theorem 1 the main tool is the following two lemas of Fujii [2].

Lemma 2. For $x>1$ and $T>T_{0}$ we have

$$
\begin{aligned}
& \sum_{0<\gamma \leq T} x^{\gamma i}=-\frac{T}{2 \pi} \frac{\Lambda(x)}{\sqrt{x}}+M(x, T)+O(\sqrt{x} \log (2 x)) \\
& +O\left(\frac{1}{\sqrt{x}} \sum_{\substack{\frac{x}{2}<n<2 x \\
n \neq x}} \Lambda(n) \cdot \min \left(T, \frac{1}{\left|\log \frac{x}{n}\right|}\right)\right) \\
& +O\left(\sqrt{x} \sqrt{\frac{\log T}{\log \log T}}\right)+O\left(x^{\frac{1}{\log \log T} \log (2 x) \frac{\log T}{\log \log T}}\right),
\end{aligned}
$$

where

$$
M(x, T)=\left\{\begin{array}{lll}
\frac{x^{i T} \log (T / 2 \pi)}{2 \pi i \log x}+O\left(\frac{1}{\log x}+\frac{1}{\log ^{2} x}\right), & \text { if } \quad \frac{1}{\log T} \ll \log x, \\
O\left(\log T \cdot \min \left(\frac{\log T}{\log x}, T \log T\right)\right), & \text { if } \quad \log x \ll \frac{1}{\log T} .
\end{array}\right.
$$

Lemma 3. Suppose that $0<\frac{2 \pi \alpha}{b} \leq Y<T, \frac{T}{2 \pi \alpha} \gg 1$ and $T>T_{0}$. Then we have for any positive $b \leq 2$,

$$
\begin{aligned}
\sum_{Y<\gamma \leq T} e\left(\frac{b \gamma}{2 \pi} \log \frac{b \gamma}{2 \pi e \alpha}\right)= & -e^{\frac{\pi i}{4}} \frac{\sqrt{\alpha}}{b} \sum_{\left(\frac{Y b}{2 \pi \alpha}\right)^{b} \leq n \leq\left(\frac{T b}{2 \pi \alpha}\right)^{b}} \Lambda(n) n^{\frac{1}{2}\left(\frac{1}{b}-1\right)} e\left(-\alpha n^{\frac{1}{b}}\right) \\
& +O\left(T^{\frac{2}{5}}\left(\frac{T}{2 \pi \alpha}\right)^{\frac{b}{2}}\right) \\
& +O\left(\left(T^{\frac{1}{2}}\left(\frac{T}{2 \pi \alpha}\right)^{-\frac{b}{2}}+Y^{\frac{1}{2}}\left(\frac{Y}{2 \pi \alpha}\right)^{-\frac{b}{2}}\right) \log \frac{T}{2 \pi \alpha}\right) \\
& +O\left(\log T \cdot \min \left(\frac{1}{\log \frac{Y}{2 \pi \alpha}}, \sqrt{\alpha}+1\right)\right) .
\end{aligned}
$$

We often will use 
Lemma 4. Assume GRH. If $0<\frac{k}{l}<1$ is rational fixed number with $(k, l)=1$, when

$$
\sum_{n \leq T} \Lambda(n) e\left(n \frac{k}{l}\right)=\frac{\mu(l)}{\varphi(l)} T+O\left(\sqrt{T} \log ^{2} T\right) .
$$

Proof. We have

$$
\sum_{n \leq T} \Lambda(n) e\left(n \frac{k}{l}\right)=\sum_{a=0}^{l-1} e\left(a \frac{k}{l}\right) \sum_{\substack{n \equiv a \bmod l \\ n \leq T}} \Lambda(n) .
$$

Then the lemma follows by (Davenport [3], Chapter 20)

$$
\sum_{\substack{n \equiv a \bmod l \\ n \leq T}} \Lambda(n)=\frac{1}{\varphi(l)} T+O\left(\sqrt{T} \log ^{2} T\right)
$$

and (Apostol [1], Exercise 2.14)

$$
\sum_{\substack{a=0 \\(a, l)=1}}^{l-1} e\left(a \frac{k}{l}\right)=\sum_{\substack{a=1 \\(a, l)=1}}^{l} e\left(a \frac{k}{l}\right)=\mu(l)
$$

\section{Proof of Theorem 1}

Using Lemma 1 we have

(4) $\sum_{0<\gamma \leq T} L\left(\frac{k}{l}, \frac{1}{2}+\gamma i\right)=\sum_{1 \leq n \leq \sqrt{\frac{T}{2 \pi}}} \sum_{2 \pi n^{2} \leq \gamma \leq T} \frac{e\left(n \frac{k}{l}\right)}{n^{\frac{1}{2}+\gamma i}}$

$$
\begin{aligned}
& +e^{\frac{\pi i}{4}} \sum_{0<\gamma \leq T} e\left(-\frac{\gamma}{2 \pi} \log \frac{\gamma}{2 \pi e}\right)\left(\frac{k}{l}\right)^{-\frac{1}{2}+\gamma i} \\
& +e^{\frac{\pi i}{4}} \sum_{1 \leq n \leq \sqrt{\frac{T}{2 \pi}}} \sum_{2 \pi n^{2} \leq \gamma \leq T} e\left(-\frac{\gamma}{2 \pi} \log \frac{\gamma}{2 \pi e}\right)\left(n+\frac{k}{l}\right)^{-\frac{1}{2}+\gamma i} \\
& +O\left(T^{\frac{3}{4}} \log T\right) \\
& =S_{1}+S_{2}+S_{3}+O\left(T^{\frac{3}{4}} \log T\right) .
\end{aligned}
$$

Term $S_{1}$. By formula (Davenport [3], Chapter 15)

$$
\sum_{0<\gamma \leq T} 1=\frac{T}{2 \pi} \log \frac{T}{2 \pi}-\frac{T}{2 \pi}+O(\log T)
$$


and Lemma 2 we get

$$
\begin{aligned}
& S_{1}=e\left(\frac{k}{l}\right) \sum_{2 \pi \leq \gamma \leq T} 1+\sum_{2 \leq n \leq \sqrt{\frac{T}{2 \pi}}} \frac{e\left(n \frac{k}{l}\right)}{n^{\frac{1}{2}}} \sum_{2 \pi n^{2} \leq \gamma \leq T} n^{-i \gamma} \\
& =e\left(\frac{k}{l}\right)\left(\frac{T}{2 \pi} \log \frac{T}{2 \pi}-\frac{T}{2 \pi}\right)+\sum_{2 \leq n \leq \sqrt{\frac{T}{2 \pi}}} \frac{e\left(n \frac{k}{l}\right)}{n^{\frac{1}{2}}}\left\{-\frac{T}{2 \pi} \frac{\Lambda(n)}{n^{\frac{1}{2}}}+n^{2} \frac{\Lambda(n)}{n^{\frac{1}{2}}}-\frac{n^{-i T} \log \frac{T}{2 \pi}}{2 \pi i \log n}\right. \\
& +\frac{n^{-2 \pi n^{2} i} \log \frac{2 \pi n^{2}}{2 \pi}}{2 \pi i \log n}+O\left(\frac{1}{\log n}+\frac{1}{\log ^{2} n}\right)+O(\sqrt{n} \log (2 n)) \\
& +O\left(\frac{1}{\sqrt{n}} \sum_{\frac{n}{2}<m<2 n, m \neq n} \Lambda(n) \cdot \min \left(T, \frac{1}{\left|\log \frac{n}{m}\right|}\right)\right) \\
& +O\left(\sqrt{n} \sqrt{\frac{\log T}{\log \log T}}\right)+O\left(n^{1 / \log \log T} \log (2 n) \frac{\log T}{\log \log T}\right) \\
& \left.-O\left(n^{1 / \log \log \left(2 \pi n^{2}\right)} \log (2 n) \frac{\log \left(2 \pi n^{2}\right)}{\log \log \left(2 \pi n^{2}\right)}\right)\right\} \text {. }
\end{aligned}
$$

Using inequalities $\Lambda(n) \leq \log n$ and $|\log (1+x)| \geq|x / 2|$ for $-\frac{1}{2} \leq x \leq 1$, similarly as in Fujii [2], we obtain

$$
\begin{aligned}
S_{1}= & e\left(\frac{k}{l}\right)\left(\frac{T}{2 \pi} \log \frac{T}{2 \pi}-\frac{T}{2 \pi}\right)-\frac{T}{2 \pi} \sum_{2 \leq n \leq \sqrt{\frac{T}{2 \pi}}} \Lambda(n) e\left(n \frac{k}{l}\right) \frac{1}{n} \\
& +\sum_{2 \leq n \leq \sqrt{\frac{T}{2 \pi}}} \Lambda(n) e\left(n \frac{k}{l}\right) n+O\left(\sqrt{T} \log ^{2} T\right)
\end{aligned}
$$

By Lemma 4 and partial summation we see that

$$
\sum_{2 \leq n \leq \sqrt{\frac{T}{2 \pi}}} \Lambda(n) e\left(n \frac{k}{l}\right) n=\frac{1}{2} \frac{\mu(l)}{\varphi(l)} \frac{T}{2 \pi}+O\left(T^{\frac{3}{4}} \log ^{2} T\right) .
$$

In (5) another sum should be calculated more precisely. Euler's theorem tells us that, for $p^{v} \| l$,

$$
p^{\varphi\left(\frac{l}{p^{v}}\right)} \equiv 1 \quad \bmod \frac{l}{p^{v}}
$$


This and the orthogonal relation of Dirichlet characters give that

$$
\begin{aligned}
& \sum_{\substack{2 \leq n \leq \sqrt{\frac{T}{2 \pi}}\\
}} \Lambda(n) e\left(n \frac{k}{l}\right) \frac{1}{n} \\
= & \sum_{\substack{2 \leq n \leq \sqrt{\frac{T}{2 \pi}} \\
(n, l)=1}} \Lambda(n) e\left(n \frac{k}{l}\right) \frac{1}{n}+\sum_{p \mid l} \log p \sum_{j=1}^{\infty} \frac{e\left(p^{j} \frac{k}{l}\right)}{p^{j}} \\
= & \frac{1}{\varphi(l)} \sum_{a=1}^{l} e\left(a \frac{k}{l}\right) \sum_{\chi} \frac{}{\chi(a)} \sum_{n \leq \sqrt{\frac{T}{2 \pi}}} \Lambda(n) \frac{1}{n} \chi(n) \\
+ & \sum_{p^{v}|| l} \frac{\log p}{1-p^{-\varphi\left(\frac{l}{p^{v}}\right)}} \sum_{j=1}^{\varphi\left(\frac{l}{p^{v}}\right)} \frac{e\left(p^{j} \frac{k}{l}\right)}{p^{j}} .
\end{aligned}
$$

If $\chi \neq \chi_{0}$ then, using the prime number theorem, we obtain

$$
\begin{aligned}
\sum_{n \leq \sqrt{\frac{T}{2 \pi}}} \Lambda(n) \frac{1}{n} \chi(n) & =\frac{L^{\prime}}{L}(1, \chi)-\sum_{n>\sqrt{\frac{T}{2 \pi}}} \Lambda(n) \frac{1}{n} \chi(n) \\
& =\frac{L^{\prime}}{L}(1, \chi)+O\left(T^{-1 / 4} \log ^{2} T\right) .
\end{aligned}
$$

If $\chi=\chi_{0}$, then by formula (3)

$$
\begin{aligned}
\sum_{n \leq \sqrt{\frac{T}{2 \pi}}} & \Lambda(n) \frac{1}{n} \chi_{0}(n)=\sum_{n=1}^{\infty} \frac{\Lambda(n) \chi_{0}(n)-1}{n}-\sum_{n>\sqrt{\frac{T}{2 \pi}}} \frac{\Lambda(n) \chi_{0}(n)-1}{n}+\sum_{n \leq \sqrt{\frac{T}{2 \pi}}} \frac{1}{n} \\
& =\sum_{n=1}^{\infty} \frac{\Lambda(n)-1}{n}-\sum_{p \mid l} \sum_{k=1}^{\infty} \frac{\log p}{p^{k}}+\frac{1}{2} \log \frac{T}{2 \pi}+\gamma(1)+O\left(T^{-1 / 4} \log ^{2} T\right) \\
& =\frac{1}{2} \log \frac{T}{2 \pi}-\sum_{p \mid l} \frac{\log p}{p-1}-\gamma(1)+O\left(T^{-1 / 4} \log ^{2} T\right) .
\end{aligned}
$$

From above, in view of

$$
\sum_{a=1}^{l} e\left(a \frac{k}{l}\right) \overline{\chi(a)}=\chi(k) \tau(\bar{\chi})
$$

it follows

$$
S_{1}=\left(e\left(\frac{k}{l}\right)-\frac{1}{2} \frac{\mu(l)}{\varphi(l)}\right) \frac{T}{2 \pi} \log \frac{T}{2 \pi}
$$

$$
+\left(-\frac{1}{\varphi(l)} \sum_{\substack{\chi \bmod _{\chi \neq} l \\ \chi \neq \chi_{0}}} \chi(k) \tau(\bar{\chi}) \frac{L^{\prime}}{L}(1, \chi)-e\left(\frac{k}{l}\right)+\left(\gamma(1)+\frac{1}{2}+\sum_{p \mid l} \frac{\log p}{p-1}\right) \frac{\mu(l)}{\varphi(l)}\right.
$$

$$
\left.-\sum_{p^{v}|| l} \frac{\log p}{1-p^{-\varphi\left(\frac{l}{p^{v}}\right)}} \sum_{j=1}^{\varphi\left(\frac{l}{p^{v}}\right)} \frac{e\left(p^{j} \frac{k}{l}\right)}{p^{j}}\right) \frac{T}{2 \pi}+O\left(T^{3 / 4} \log ^{2} T\right) .
$$


Term $S_{2}$. Using Lemmas 3 and 4 we get

$$
\begin{aligned}
S_{2} & =e^{\frac{\pi i}{4}}\left(\frac{k}{l}\right)^{-\frac{1}{2}} \sum_{6 \pi \frac{k}{l} \leq \gamma \leq T} e\left(-\frac{\gamma}{2 \pi} \log \frac{\gamma}{2 \pi e \frac{k}{l}}\right)+O(1) \\
& =-\sum_{1 \leq n \leq \frac{T}{2 \pi \frac{k}{l}}} \Lambda(n) e\left(n \frac{k}{l}\right)+O\left(T^{\frac{9}{10}}\right) \\
& =-\frac{l}{k} \frac{\mu(l)}{\varphi(l)} \frac{T}{2 \pi}+O\left(T^{\frac{9}{10}}\right) .
\end{aligned}
$$

Term $S_{3}$. Again we use Lemmas 3 and 4 .

$$
\begin{aligned}
S_{3} & =e^{\frac{\pi i}{4}} \sum_{1 \leq n \leq \sqrt{\frac{T}{2 \pi}}}\left(n+\frac{k}{l}\right)^{-\frac{1}{2}} \sum_{2 \pi n^{2} \leq \gamma \leq T} e\left(-\frac{\gamma}{2 \pi} \log \frac{\gamma}{2 \pi e\left(n+\frac{k}{l}\right)}\right) \\
& =-\sum_{1 \leq n \leq \sqrt{\frac{T}{2 \pi}}} \sum_{\frac{n^{2}}{n+\frac{k}{l}} \leq m \leq \frac{T}{2 \pi\left(n+\frac{k}{l}\right)}} \Lambda(m) e\left(m \frac{k}{l}\right)+O\left(T^{\frac{9}{10}} \log T\right) \\
& =-\frac{\mu(l)}{\varphi(l)} \sum_{1 \leq n \leq \sqrt{\frac{T}{2 \pi}}}\left(\frac{T}{2 \pi\left(n+\frac{k}{l}\right)}-\frac{n^{2}}{n+\frac{k}{l}}\right)+O\left(T^{\frac{9}{10}} \log T\right) \\
& =-\frac{\mu(l)}{\varphi(l)} \sum_{1 \leq n \leq \sqrt{\frac{T}{2 \pi}}}\left(\frac{T}{2 \pi\left(n+\frac{k}{l}\right)}-\frac{\left(\frac{k}{l}\right)^{2}}{n+\frac{k}{l}}-\left(n-\frac{k}{l}\right)\right)+O\left(T^{\frac{9}{10}} \log T\right) .
\end{aligned}
$$

Then in view of (2) (note that in the definition of the generalized Euler constant the summation starts from zero) we have

$$
S_{3}=-\frac{1}{2} \frac{\mu(l)}{\varphi(l)} \frac{T}{2 \pi} \log \frac{T}{2 \pi}+\left(\frac{l}{k}-\gamma\left(\frac{k}{l}\right)+\frac{1}{2}\right) \frac{\mu(l)}{\varphi(l)} \frac{T}{2 \pi}+O\left(T^{\frac{9}{10}} \log T\right) .
$$

Now the theorem follows by (4), (7), (9), and (10).

\section{REFERENCES}

[1] T. M. Apostol, Introduction to Analytic Number Theory, Springer-Verlag, New York, 1976

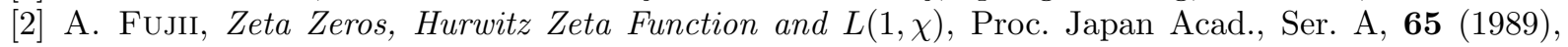
$139-142$

[3] G. Davenport, Multiplicative Number Theory. Markham, Chicago, 1967

[4] R. Garunkštis, A. Laurinčikas, and J. Steuding, An approximate functional equation for the Lerch zeta-function, Math. Notes, 74 (2003), 469-476

[5] R. Garunkštis and J. Steuding, On the Distribution of Zeros of the Hurwitz Zeta-function, Math. Comput., 76 (2007), 323-337

[6] J. Steuding, On the value-distribution of the Hurwitz zeta-function at the nontrivial zeros of the Riemann zeta-function, Abhdlg, Math. Sem. Uni. Hamburg, 71 (2001), 113-121

[7] J. Steuding, Dirichlet series associated to periodic Arithmetic Functions and the zeros of Dirichlet L-functions. Anal. Probab. Methods Number Theory, (2002), 282-296 
Ramūnas Garunkštis

Department of Mathematics and Informatics

Vilnius University

Naugarduko 24

2600 Vilnius

Lithuania

ramunas.garunkstis@maf.vu.lt
Justas Kalpokas

Department of Mathematics

and Informatics

Vilnius University

Naugarduko 24

2600 Vilnius

Lithuania

justas.kalpokas@maf.vu.lt 